\title{
Prevalência e severidade das alteraçôes oclusais em escolares de 12 a 15 anos de Salvador - BA, 2004
}

\author{
Laira Sá Lopes ${ }^{I}$ \\ Maria Cristina Teixeira Cangussu
}

\begin{abstract}
Resumo
Apesar de haver uma evidente percepção do crescimento da demanda por tratamento ortodôntico na sociedade contemporânea, poucos ainda são os dados epidemiológicos sobre esse problema de saúde. Este trabalho buscou determinar a prevalência e a severidade de alterações oclusais em escolares de 12 a 15 anos de Salvador - Bahia, através de um levantamento epidemiológico de base populacional, utilizando-se como indicadores a classificação de Angle, o Índice de Estética Dentária (IED) e o registro dos desvios morfológicos dentários e faciais. Foram examinados por seis examinadores previamente calibrados 2100 escolares na faixa etária de 12 a 15 anos, selecionados através de uma amostra aleatória, representativa para o município de Salvador nessa faixa etária. Dentre os adolescentes, 899 eram do sexo masculino, 1211 do sexo feminino, e a etnia predominante foi a parda (70,76\%). A média do IED neste estudo foi de 26, 06, com um desvio padrão de 7,67. Segundo as categorias de necessidade de tratamento, foram encontrados os seguintes valores: nenhum ou pouco, 54,31\%; tratamento eletivo, 23,22\%; tratamento altamente desejável, 11,33\%; e tratamento mandatório, 11,14\%. De acordo com a classificação de Angle, a maloclusão mais encontrada foi a Classe I (39,67\%), seguida das Classes III e II (23,55\% e 19,00\%) respectivamente. Além disso, os desvios morfológicos clínicos de maior ocorrência nessa população foram o desvio de linha média e a sobremordida.
\end{abstract}

Palavras-chave: má oclusão; epidemiologia; odontologia em saúde coletiva.

\section{INTRODUÇÃO}

Inúmeros pesquisadores, nos últimos anos, têm ressaltado a necessidade de se conhecer a situação epidemiológica das oclusopatias, de forma a permitir o direcionamento de atitudes preventivas ou curativas pelo setor saúde, visto que parece ser crescente a demanda por tratamento ortodôntico na sociedade contemporânea.

Entretanto, o grande número de indicadores tem tornado difícil a comparação dos re- sultados obtidos em diferentes regiōes, além de, em muitas regióes, não existirem dados epidemiológicos sobre esse problema de saúde.

Dentre as classificações mais utilizadas para o diagnóstico das alteraçôes oclusais, têmse a de Angle, dividida em quatro categorias; o registro individual de desvios morfológicos, como as mordidas cruzadas posteriores e anteriores, a mordida aberta anterior, o apinhamento dentário, entre outros. Mais recentemente, na

\footnotetext{
${ }^{1}$ Bolsista de Iniciação Científica CNPQ-UFBA. Faculdade de Odontologia. UFBA. Salvador - BA

${ }^{2}$ Professora Adjunto - Departamento de Odontologia Social e Pediátrica. Faculdade de Odontologia. UFBA. Salvador - BA

Correspondência para / Correspondence to:

Maria Cristina Teixeira Cangussu

Departamento de Odontologia Social e Pediátrica. UFBA

Rua Araújo Pinho 62- Canela.

41.110-150 Salvador-Bahia-Brasil.

E-mail: cangussu@ufba.br.
} 
tentativa de uniformizar os dados epidemiológicos produzidos sobre as alteraçôes oclusais, a Organização Mundial da Saúde adotou, em 1997, o Dental Aesthetic Index (DAI), que registra dez condições a serem observadas nos indivíduos, referentes a dentição, espaço e oclusão (ORGANIZAÇÃO MUNDIAL DA SAÚDE, 1997).

A classificação de Angle, embora não tenha sido desenvolvida como um indicador epidemiológico, tem sido muito utilizada em estudos internacionais e nacionais, provavelmente por ser de fácil aplicação, abranger quase todos os tipos de oclusopatias e ser de grande conhecimento dos profissionais da odontologia. Através dela, é possível verificar, na população, o número total de indivíduos cujas relações dento-maxilares se apresentam em desacordo com uma oclusão considerada normal, tomando como referência a posição dos primeiros molares permanentes no arco dentário.

Vários estudos publicados no país utilizaram esse indicador. Silva e Araújo (1983) examinaram 600 crianças entre 5 e 7 anos, de escolas da Ilha do Governador, no Rio de Janeiro, e classificaram $30,8 \%$ das crianças como portadoras de oclusão normal, $54,7 \%$ das crianças com Classe I, 13\% com Classe II e 13\% com Classe III. Silva Filho, Freitas e Cavassan (1989), analisando 2416 escolares de 7 a 11 anos na cidade de Bauru, em São Paulo, detectaram apenas $11,47 \%$ de crianças com oclusão normal, sendo que a categoria predominante foi a Classe I, com 55\% das crianças, seguida das Classes II (42\%) e III (3\%) respectivamente.

$\mathrm{Na}$ década de 90, os estudos publicados na literatura registram números ainda mais alarmantes quanto à prevalência de oclusopatias. Biscaro, Pereira e Magnani (1994), examinando 891 crianças de 7 a 12 anos em Piracicaba, São Paulo, identificaram $97,7 \%$ de desvios na oclusão, sendo que, desses, $68,8 \%$ eram referentes à Classe I, $17,8 \%$ à Classe II e 5,2\% à Classe III. Perin (1997) analisou a prevalência de má oclusão em crianças de 12 anos de Lins e Guaiçara, São Paulo, num total de 272 crianças. Na cidade de Lins, foi descrita uma prevalência de $81,30 \%$ de oclusopatias e, em Guaiçara, 90,60\%, predominando, em ambas as regiōes, a Classe I de Angle. Resultado similar também foi descrito por Estacia (2000) em escolares de 12 a 15 anos de Passo Fundo, Rio Grande do Sul, que encontrou 8,26\% de oclusão normal, 50,51\% de Classe I; 38,54\% da Classe II e $2,69 \%$ da Classe III.

Entretanto, vários autores (SILVA FILHO; FREITAS; CAVASSAN, 1989; PIRES; ROCHA; CANGUSSU, 2001; ESTACIA, 2000; QUELUZ; GIMENEZ, 2000) destacaram a necessidade de, além do registro da classificação de Angle, detectar-se o tipo de desvio morfológico presente, de forma a facilitar a comunicação profissional e a determinação da necessidade de tratamento de uma determinada população. Assim, dentre as principais características clínicas investigadas, encontram-se: as mordidas cruzadas anteriores e posteriores (ESTACIA, 2000; BISCARO; PEREIRA; MAGNANI, 1994; SILVA FILHO; FREITAS; CAVASSAN, 1989); o trespasse horizontal e vertical dos incisivos anteriores, incluindo a mordida aberta anterior (ESTACIA, 2000; QUELUZ; GIMENEZ, 2000; SILVA FILHO; FREITAS; CAVASSAN, 1989); postura labial (ESTACIA, 2000) e inserção do freio labial; e perda precoce de dentes decíduos (SILVA FILHO; FREITAS; CAVASSAN, 1989).

De forma a resgatar o uso de indicadores epidemiológicos para mensurar as oclusopatias, a Organização Mundial da Saúde (1997) propõe, no seu último manual, o uso do Índice de Estética Dentária (IED), constituído como um parâmetro matemático de indicadores clínicos e estéticos (definidos a partir do impacto que as diversas características oclusais provocam no convívio social e no desenvolvimento psicológico) para a definição de prioridade da necessidade de tratamento ortodôntico (JENNY et al., 1991). Construiuse, então, uma equação matemática que contém 10 componentes, com seus respectivos coeficientes: total de dentes anteriores ausentes, apinhamento no segmento incisal, espaçamento no segmento incisal, diastema maxilar, discrepâncias maxilar e mandibular, overjet maxilar e mandibular e relação molar, e um valor numérico constante de 13. 
A partir do valor obtido no IED, são classificadas quatro indicaçôes de necessidade de tratamento: de 15 a 25 , que indicaria a presença de alteração oclusal sem relevância clínica; de 26 a 31, em que o tratamento é necessário, mas não premente; de 32 a 35 , em que o tratamento é muito desejável; e acima de 36 , em que o tratamento seria considerado mandatório (ORGANIZAÇĀO MUNDIAL DA SAÚDE, 1997).

Poucos ainda são os trabalhos publicados que utilizam o IED no mundo (JENNY et al., 1991; ANSAI et al., 1993; ESTIOKO; WRIGHT; MORGAN, 1994; KATOH et al., 1998; OTUYEMI et al., 1999; JOHNSON; HARKNESS, 2000; ONYEASO, 2003), pois os autores têm encontrado diferentes necessidades de intervenção ortodôntica em diferentes locais, atribuindo-as a características étnicas de cada região, cuja influência é decisiva nas alterações oclusais.

No Brasil, escassos estudos que utilizam o IED foram identificados na literatura científica. Narvai e colaboradores (2003) publicaram o resultado de um levantamento realizado no estado de São Paulo, segundo o qual, aos 12 anos de idade, $64 \%$ dos escolares foram classificados sem anormalidade ou com má oclusão leve; $18 \%$ com alguma má oclusão definida, e $9 \%$ de alterações oclusais severas e muito severas ou incapacitantes. Dentre as características clínicas mais presentes, registrou-se o apinhamento dentário, em 35\% dos escolares. Peres, Traebert e Marcenes (2002) realizaram, em Porto Alegre, Rio Grande do Sul, um estudo com escolares de 14 a 18 anos, no qual a prevalência de algum tipo de oclusopatia foi de $71,3 \%$. Perin (2002) também analisou as oclusopatias em 734 escolares de 12 anos de Lins, São Paulo, registrando 65,26\% das crianças com valores do IED abaixo de 25 ; entre 26 e $30,12,81 \%$ das crianças; entre 31 e 35 , 10,9\%; e acima de $36,11 \%$ da amostra. Além desses trabalhos, o SB Brasil, levantamento epidemiológico das condições de saúde bucal da população brasileira, realizado nos anos de 2002 e 2003, optou pelo uso desse indicador.

Entretanto, diversos autores têm identificado algumas limitaçōes do Índice de Estética Dentária, por sua incapacidade de identifi- car alterações clínicas específicas e de grande necessidade de intervenção ortodôntica, como a sobremordida, que pode provocar lesões gengivais ou no palato, a mordida cruzada e a mordida aberta posterior, bem como a dificuldade da operacionalização do índice em crianças na dentição mista (ANSAI et al., 1993; PERIN, 2002).

Nesse sentido, julga-se oportuna a realização de um levantamento epidemiológico de base populacional em Salvador, utilizando-se os indicadores mais comuns descritos na literatura para o diagnóstico das alterações oclusais, com o objetivo de diagnosticar, de forma representativa na população escolar, a prevalência de oclusopatias, bem como testar a adequação do IED para o registro da necessidade de tratamento desse grupo.

\section{MATERIAIS E MÉTODOS}

O desenho proposto para este estudo foi o transversal, e a população de referência constituiu-se de escolares de 12 anos a 15 anos $(\mathrm{N}=220.300)$, de ambos os sexos, regularmente matriculados em escolas de $1^{\circ}$. e $2^{\circ}$. graus, públicas e privadas, de Salvador, Bahia, no ano de 2004. Optou-se pela obtenção de uma amostra probabilística em múltiplo estágio, cujas unidades amostrais primárias representaram o cadastro de escolas públicas e particulares da cidade, fornecido pelo Ministério da Educação (MEC). Foram sorteados 40 estabelecimentos de ensino, $10 \%$ do total de 398 escolas, obedecendo-se a proporcionalidade entre estudantes de escolas públicas e privadas. Posteriormente, a seleção das crianças no ambiente escolar se deu por amostragem sistemática.

Para o cálculo da amostra, foram usados os dados populacionais mais recentes disponíveis da proporção de oclusopatias aos 12 anos de idade, descritos nos estudos nacionais (NARVAI et al., 2003; PERIN, 2002), considerando-se a representatividade para uma prevalência estimada de $10 \%$ na categoria de maior severidade do IED. O nível de significância adotado foi de $1 \%$, e o poder do teste foi de $90 \%$. A distribuição Qui-quadrado foi considerada a mais pertinente para o cálculo 
da amostra, utilizando-se o método da amostragem para proporções. O número amostral mínimo obtido foi de 386 crianças e estimando-se uma perda de $30 \%$, totalizou-se então um tamanho de amostra de 500 crianças em cada faixa etária, com um total de 2.000 escolares.

O desenvolvimento deste estudo seguiu os requisitos da Resolução 196/96 do Conselho Nacional de Saúde, com parecer favorável do Comitê de Ética do Instituto de Saúde Coletiva da UFBA.

A coleta de dados foi realizada em ambiente escolar, por 6 examinadores previamente calibrados (concordância geral $84 \%$ ), utilizando-se uma ficha desenvolvida para este estudo. Foram obtidas as seguintes informaçōes:

- Dados gerais: nome, data de nascimento, endereço de residência, nome da escola, tipo de financiamento da escola (pública, privada), escolaridade dos pais, etnia, uso anterior ou atual de aparelho ortodôntico, história de hábitos deletérios.

- Exame clínico: foram inspecionadas as condiçōes oclusais segundo a classificação de Angle (FERREIRA, 1999), o Índice de Estética Dentária (ORGANIZAÇĀO MUNDIAL DA SAÚDE, 1997) e a presença de desvios morfológicos não identificados pelos indicadores anteriores.

Os dados foram digitados e armazenados num banco de dados criado no programa MINITAB, onde se procedeu a análise. Conduziu-se a caracterização da população de estudo e das alteraçôes oclusais no município, apresentadas segundo condição de necessidade de tratamento do IED; proporção da amostra segundo a classificação de Angle; desvios morfológicos clínicos de maior ocorrência. O teste estatístico do Qui-quadrado com um nível de significância de $5 \%$ foi utilizado para identificar diferenças entre as prevalências das alterações oclusais, segundo os indicadores utilizados.

\section{RESULTADOS}

Foram entrevistados e examinados 2.100 adolescentes de 12 a 15 anos de idade de Salva- dor, Bahia. Predominaram, na amostra: etnia parda, alunos de escolas públicas, do sexo feminino, provenientes de famílias em que os pais possuíam pouca escolaridade. Dentre os hábitos deletérios associados à presença de alteraçôes oclusais, identificou-se em maior proporção, o uso de chupeta, a sucção do dedo após os cinco anos de idade, a onicofagia, a presença de respiração oral, interposição lingual e o uso de mamadeira por mais de 5 anos (TABELA 1).

Tabela 1-Caracterização da população de estudo segundo idade, sexo, etnia, tipo de eestabelecimento de ensino, escolaridade dos pais e hábitos deletérios identificados. Salvador - BA, 2004 .

\begin{tabular}{lcc}
\hline Variáveis & $\mathrm{n}$ & $\%$ \\
\hline Idade (em anos) & 521 & 24,69 \\
12 & 543 & 25,73 \\
13 & 535 & 25,36 \\
14 & 511 & 24,22 \\
15 & & \\
Etnia & 131 & 6,21 \\
Branca e outras & 486 & 23,03 \\
Negra & 1493 & 70,76 \\
Parda & & \\
Estabelecimento de ensino & 1753 & 83,08 \\
Público & 357 & 16,92 \\
$\quad$ Privado & & \\
Escolaridade dos pais & 180 & 8,57 \\
Superior incompleto ou mais & 747 & 35,57 \\
Fundamental completo a médio completo & 1183 & 55,86 \\
Fundamental incompleto ou menos & & \\
\hline
\end{tabular}

Os dados obtidos através do Índice de Estática Dentária apontam que 54,24\% dos adolescentes se enquadram na categoria sem necessidade de tratamento. As demais categorias tiveram as seguintes expressōes: tratamento eletivo, 26,29\%; tratamento desejável, 8,29\%; e tratamento mandatório, $11,18 \%$. Dentre os indivíduos examinados, $82,23 \%$ apresentaram desvios na relação molar, segundo a classificação de Angle, com 39,67\% na Classe I; 19,01\% na Classe II e 23,55\%, Classe III. Na distribuição 
dos desvios morfológicos, o desvio de linha média teve a maior ocorrência $(30,33 \%)$, seguido da sobremordida $(17,48 \%)$ e mordida cruzada posterior $(13,14 \%)$ (TABELA 2).

Os componentes do Índice de Estética Dentária que tiveram maior freqüência nas categorias de maior necessidade de tratamento foram o overjet maxilar $(91,95 \%$ tratamento

Tabela 2 - Condição oclusal dos 2.100 adolescentes de 12 a 15 anos, segundo o IED, a classificação de Angle (molar e canina) e a presença de outros desvios morfológicos. Salvador - BA, 2004.

\begin{tabular}{|c|c|c|}
\hline Indicador & $\mathrm{N}$ & $\%$ \\
\hline \multicolumn{3}{|l|}{ IED } \\
\hline-25 sem necessidade tratamento & 1139 & 54,24 \\
\hline $26|-| 31$ tratamento eletivo & 552 & 26,29 \\
\hline $32|-| 35$ tratamento desejável & 174 & 08,29 \\
\hline$>35$ tratamento mandatório & 235 & 11,18 \\
\hline \multicolumn{3}{|l|}{ Angle - relação molar } \\
\hline Normal & 375 & 17,86 \\
\hline Classe I & 837 & 39,86 \\
\hline Classe II & 401 & 19,10 \\
\hline Classe III & 497 & 23,18 \\
\hline \multicolumn{3}{|l|}{ Angle - relaçăo canina } \\
\hline Normal & 504 & 24,00 \\
\hline Classe I & 743 & 35,38 \\
\hline Classe II & 442 & 21,05 \\
\hline Classe III & 421 & 19,57 \\
\hline \multicolumn{3}{|l|}{ Outros desvios morfológicos } \\
\hline Mordida cruzada posterior & 276 & 13,14 \\
\hline Mordida aberta posterior & 178 & 08,48 \\
\hline Sobremordida & 367 & 17,48 \\
\hline Desvio de linha média & 637 & 30,33 \\
\hline $\begin{array}{l}\text { Outros (hipodontias, anodon tias, fraturas } \\
\text { severas) }\end{array}$ & 265 & 12,62 \\
\hline
\end{tabular}

desejável e 91,06\% tratamento mandatório), seguido do apinhamento dentário anterior $(71,84 \%$ e $75,14 \%)$ e o desalinhamento maxilar $(60,92 \%$ e $70,64 \%)$. Nota-se que a expressão dos desvios na relação molar antero-posterior aumenta à medida que há maior necessidade de tratamento (TABELA 3).

\section{DISCUSSÃO}

Observou-se uma predominância, neste estudo, de adolescentes sem necessidade de
Tabela 3 - Composição percentual do IED em adolescentes de 12 a 15 anos de idade, segundo necessidade de tratamento. Salvador - BA, 2004.

\begin{tabular}{|c|c|c|c|c|}
\hline Condição & $\begin{array}{l}\text { Normal } \\
\mathrm{n}=1139\end{array}$ & $\begin{array}{c}\text { Tratamento } \\
\text { eletivo } \\
\mathrm{n}=552\end{array}$ & $\begin{array}{c}\text { Tratamento } \\
\text { desejável } \\
n=174\end{array}$ & $\begin{array}{c}\text { Tratamento } \\
\text { mandatório } \\
\mathrm{n}=235\end{array}$ \\
\hline \multicolumn{5}{|l|}{ Ausência dentária anterior } \\
\hline Nenhuma & 98,77 & 91,67 & 84,48 & 83,40 \\
\hline 1 ou mais & 1,23 & 8,33 & 15,52 & 16,60 \\
\hline \multicolumn{5}{|l|}{ Apinhamento anterior } \\
\hline Nenhum & 54,70 & 32,25 & 28,16 & 24,68 \\
\hline 1 ou mais segmentos & 45,30 & 67,75 & 71,84 & 75,14 \\
\hline \multicolumn{5}{|l|}{ Espaçamento anterior } \\
\hline Nenhum & 70,76 & 55,07 & 51,72 & 58,72 \\
\hline 1 ou mais segmentos & 29,24 & 44,93 & 48,28 & 41,23 \\
\hline \multicolumn{5}{|l|}{ Diastema } \\
\hline Ausente & 86,92 & 70,29 & 65,52 & 65,53 \\
\hline $1 \mathrm{~mm}$ ou mais & 13,08 & 29,71 & 34,48 & 34,47 \\
\hline \multicolumn{5}{|l|}{ Desalinhamento maxilar } \\
\hline Nenhum & 68,57 & 48,01 & 39,08 & 29,36 \\
\hline $1 \mathrm{~mm}$ ou mais & 31,43 & 51,99 & 60,92 & 70,64 \\
\hline \multicolumn{5}{|c|}{ Desalinhamento mandibular } \\
\hline Nenhum & 63,74 & 41,30 & 39,66 & 32,77 \\
\hline $1 \mathrm{~mm}$ ou mais & 36,26 & 58,70 & 60,34 & 67,23 \\
\hline \multicolumn{5}{|l|}{ Overjet maxilar } \\
\hline Até $2 \mathrm{~mm}$ & 10,97 & 7,97 & 8,05 & 8,94 \\
\hline $3 \mathrm{~mm}$ ou mais & 89,03 & 92,03 & 91,95 & 91,06 \\
\hline \multicolumn{5}{|l|}{ Overjet mandibular } \\
\hline Nenhum & 98,95 & 95,47 & 91,95 & 82,55 \\
\hline $1 \mathrm{~mm}$ ou mais & 1,05 & 4,53 & 8,05 & 14,45 \\
\hline \multicolumn{5}{|l|}{ Mordida aberta anterior } \\
\hline Ausente & 97,54 & 92,39 & 78,16 & 60,43 \\
\hline $1 \mathrm{~mm}$ ou mais & 2,46 & 7,61 & 21,84 & 39,57 \\
\hline \multicolumn{5}{|l|}{ Relação molar } \\
\hline Normal & 69,80 & 38,04 & 29,89 & 19,15 \\
\hline 1 cúspide & 21,69 & 36,23 & 33,91 & 32,77 \\
\hline 2 ou mais cúspides & 8,51 & 25,72 & 36,21 & 48,09 \\
\hline
\end{tabular}

tratamento ortodôntico segundo o Índice de Estática Dentária, seguidos daqueles que requerem tratamento eletivo, mandatório e desejável, respectivamente. Esses resultados se assemelham aos encontrados por Estioko, Wright e Morgan (1994), Otuyemi e colaboradores (1999), Narvai e colaboradores (2003) e Perin (2002), nos quais o percentual de crianças sem anormalidades e com má oclusão leve foi maior que os percentuais de má oclusão definida, severa e 
muito severa. Estudos como os de Johnson e Harkness (2000) e de Peres, Traebert e Marcenes (2002), de modo contrário, apontam um maior número de ocorrências de má oclusão definida, severa e muito severa, em relação à categoria que dispensa tratamento, reforçando a necessidade de se analisar, em âmbito local, as diferentes necessidades de tratamento entre populações.

Silva e Araújo (1983) apontam que a prevalência de má oclusão parece ser menor em grupos primitivos e em grupos étnicos puros, e aparentemente maior em populações constituídas de várias descendências raciais. A população brasileira, especificamente do município de Salvador, tem como característica uma grande heterogeneidade étnica, conseqüência da forte miscigenação de indivíduos de diferentes características raciais, o que pode ter contribuído para uma prevalência significativa de alterações oclusais.

Em relação às condições de espaço e oclusão, o overjet maxilar, o apinhamento dentário anterior e o desalinhamento maxilar foram os componentes do Índice de Estética Dentária mais expressivos para a categoria de maior severidade. Nos estudos de Perin (2002) e Estioko, Wright e Morgan (1994), o overjet maxilar anterior também foi a alteração observada com maior freqüência na composição desse índice. Diferentes resultados foram encontrados por Otuyemi e colaboradores (1999) e Narvai e colaboradores (2003). A maioria dos adolescentes examinados apresentou relação molar antero-posterior normal, assim como nos trabalhos de Estioko, Wright e Morgan (1994) e de Otuyemi e colaboradores (1999). No estudo de Johnson e Harkness (2000), entretanto, o desvio da relação molar foi mais prevalente que a relação molar antero-posterior normal.

Autores como Ansai e colaboradores (1993), Otuyemi e colaboradores (1999), e Perin (2002) consideram o registro de alterações importantes, como a sobremordida, mordida aberta posterior, mordida cruzada posterior e ausência de dentes posteriores como uma das principais limitaçôes do Índice de Estética Dentária. Além disso, outro fator que o limita é a sua inadequação para a dentição decídua e mista, o que dificulta a prevenção e o tratamento precoce dos casos de má oclusão. Deve-se salientar que ainda não é consenso a utilização sistemática do Índice de Estética dentária para definir a necessidade de tratamento na população brasileira. Como ressalvado por Peres, Traebert e Marcenes (2002), os critérios de necessidade de tratamento devem estar adequados à percepção individual da população, existindo ainda poucos estudos no Brasil sobre esse assunto.

No presente estudo, os desvios morfológicos de maior ocorrência foram o desvio de linha média, seguido da sobremordida e mordida cruzada. Outros trabalhos também relatam tais desvios como mais freqüentes. Biscaro, Pereira e Magnani (1994), examinando 891 crianças de 7 a12 anos, observou 22,1\% de mordida cruzada e $15,3 \%$ de overbite. No estudo de Silva Filho, Freitas e Cangussu (1989), foram examinadas 2416 crianças de 7 a 11 anos, constatando-se $19,86 \%$ de sobremordida e $18,2 \%$ de mordida cruzada posterior.

Segundo a classificação de Angle, dentre os indivíduos examinados, $82,23 \%$ apresentaram desvios na relação molar, com uma maior proporção para a Classe I, seguida da Classe III. Silva Filho, Freitas e Cangussu (1989), num levantamento realizado na cidade de Bauru, São Paulo, ao examinar 2416 crianças entre 7 e 11 anos, encontrou apenas $11,47 \%$ de oclusão normal; 55,00\% Classe I; 42,00\% Classe II; 3,00\% Classe III. Do mesmo modo, Biscaro, Pereira e Magnani (1994), na cidade de Piracicaba, em São Paulo, de um total de 891 crianças, classificaram como Classe I $68,8 \%$ das crianças entre 7 e 12 anos do estudo; 17,8\% Classe II; 5,2\% Classe III. Estacia (2000), na cidade de Passo Fundo, Rio Grande do Sul, examinou 1562 escolares de 12 a 15 anos, encontrando 8,26\% de oclusão normal, 50,51\% Classe I, 38,54\% Classe II e 2,69\% Classe III. Esses dados fornecem percentuais bem menores de indivíduos classificados como Classe III, quando comparados aos encontrados neste estudo, o que pode indicar características demográficas específicas para a população de município com grande miscige- 
nação étnica, o que pode contribuir significativamente nos padrões esqueléticos e padrōes faciais.

Pontuou-se claramente a identificação de uma alta prevalência de má oclusão, a partir da classificação de Angle. Contudo, assim como já fora apontado por autores como Biscaro, Pereira e Magnani (1994) e Perin (2002), essa constatação pouco contribuiu para definir a prioridade de tratamento ortodôntico, por não caracterizar as alteraçóes de forma que permitiriam a classificação dos indivíduos ,a fim de estabelecer pontos de corte da severidade.
Em busca do estabelecimento de políticas adequadas para o tratamento da má oclusão no País, é nítida a preocupação atual sobre esse tema na literatura. Porém ainda são poucos os estudos com utilização de indicadores que estabeleçam parâmetros de severidade, como o Índice de Estética Dentária. Tendo em vista o impacto que a má oclusão pode representar na qualidade de vida dos indivíduos, a realização de mais estudos com utilização desse índice é essencial na implementação de adequaçóes que tornem esse indicador adequado para a população brasileira, favorecendo a comparação com os resultados obtidos em regiōes distintas.

\section{Prevalence and severity of malocclusion in schoolchildren from 12 to 15-years- old of Salvador-BA, 2004}

\section{Abstract}

Although an evident perception of growth of the demand for orthodontic treatment in the society contemporary, there are few epidemiologic data on this health problem. The aim of this work was to determine the prevalence and severity of maloocclusois in schoolchildren of 12 the 15 years of Salvador-BA, through a survey of population base using as indicating the classification of Angle, the Dental Aesthetic Index (DAI) and the register of dental and face morphologic problems. The 2100 adolescents from 12 to 15-years-old, chosen through a random representative sample for the city of Salvador-BA had been examined by six examiners previously calibrated. Amongst the adolescents, 899 were of the boys, 1211 of girls, and the predominant ethnic group was mulatto (70,76\%). The average of DAI in this study was of 26.06 with a standard deviation of 7.67. According to categories of treatment necessity had been found the following values: "none" - 54.31\%, "elective treatment"-23.22\%, "highly desirable treatment" $11.33 \%$ and "mandatory treatment" - 11.14\%. In accordance with the classification of Angle, the most found malocclusion was Class I (39.67\%), followed of Class III and II (23.55\% and 19.00\%) respectively. Moreover, the clinical morphologic with bigger occurrence in this population had been the central incisors deviation and overcross.

Keywords: malocclusion-epidemiology; public health dentistry.

\section{REFERÊNCIAS}

ANSAI, T. et al. Prevalence of malocclusion in high school students in Japan according to the Dental Aesthetic Index. Community Dent. Oral Epidemiol., Copenhagen, v.21, p.303-305, June 1993.

BISCARO, S.L.; PEREIRA, A.C.; MAGNANI, M.B.B.A. Avaliação da prevalência de má- oclusão em escolares de Piracicaba-SP na faixa etária de 7 a 12 anos. R. Odontopediatr., Ribeirão Preto, v.3, n.3, p.145-153, set. 1994.

ESTACIA, A. Características das oclusões encontradas em escolares de 12 a 15 anos na cidade de Passo Fundo, RS. 2000. Dissertação (Mestrado em Ortodontia)- Faculdade de Odon- 
tologia, Universidade Federal do Rio de Janeiro, Rio de Janeiro, 2000.

ESTIOKO, L.J.; WRIGHT, F.A.C.; MORGAN, M.V. Orthodontic treatment need of secondary schoolchildren in Heidelberg, Victoria: an epidemioloic study using the Dental Aesthetic Index. Community Dent. Health, Lowestoft, v.11, p.147-151, June 1994.

FERREIRA, F.V. Classificação das más oclusões. In: _-___- Ortodontia: diagnóstico e planejamento clínico. 3.ed. São Paulo: Artes Médicas, 1999. p.97-114.

JENNY, J. et al. Differences in need of orthodontic treatment between native Americans and the general population based on DAI scores. J. Public Health Dent., Raleigh, v.51, n.4, p.234-238, Sept. 1991.

JOHNSON, M.; HARKNESS, M. Prevalence of malocclusion and orthodontic treatment nedd in 10-year-old New Zealand children. Aust. Orthod. J., Brisbane, v.16, p.1-8, Mar. 2000.

KATOH, Y. et al. A comparation of DAI scores and characteristics of occlusal traits in three ethinic groups of Asian origin. Int. Dent. J., London, v.48, p.405-411, Sept. 1998.

NARVAI, P.C .et al. Levantamento epidemiológico em saúde bucal: estado de São Paulo, 1998. Disponível em: < $\underline{\text { http:// }}$ www.saude.sp.gov.br/html/fr sbucal.htm> Acesso em: 21 dez. 2003.

ONYEASO, C.O. An assessment of relationship between self-esteem, orthodontic concern and Dental Aesthetic Index (DAI) scores among secondary school students in Ibadan, Nigeria. Int. Dent. J., London, v.53, p.79-84, Mar. 2003.

ORGANIZAÇÃO MUNDIAL DA SAÚDE. Oral health surveys: basic methods. Geneva: World Health Organization, 1997.

OTUYEMI, O.D. et al. Malocclusion and orthodontic treatment need of secondary school students in Nigéria according to the Dental Aesthetic Index (DAI). Int. Dent. J., London, v.49, p.203-210, June 1999.

PERES, K.G.; TRAEBERT, E.A.S.; MARCENES W. Diferenças entre autopercepção e critérios normativos na identificação das oclusopatias. R. Saúde Públ., São Paulo, v.36, n.2, p.230-236, Mar. 2002.

PERIN, P.C. Influência da fluoretação da água de abastecimento público na prevalência de cárie e maloclusão. 1997. Dissertação (Mestrado em Odontologia Preventiva e Social)- Faculdade de Odontologia, Universidade Estadual Paulista, Araçatuba, SP, 1997.

PERIN, P.C. Prevalência e má-oclusão e necessidade de tratamento ortodôntico, comparando a classificação de Angle e o Índice de Estética Dentária, na cidade de Lins/SP. 2002. Tese (Doutorado em Odontologia Preventiva e Social)- Faculdade de Odontologia, Universidade Estadual de São Paulo, Araçatuba, SP, 2002.

PIRES, D.M.; ROCHA, M.C.B.S.; CANGUSSU, M.C.T. Prevalência de oclusopatias na dentadura mista em escolares- SalvadorBA. R. Bras. Odontol., Rio de Janeiro, v.58, n.6, p.414-417, Dez. 2001.

QUELUZ, D.P.; GIMENEZ, C.M.M. Prevalência do trespasse horizontal e vertical dos incisivos em escolares. R. Fac. Odontol. Passo Fundo, Passo Fundo, v.5, n.1, p.27-31, jun. 2000.

SILVA, C.H.T.; ARAÚJO, T.M. Prevalência de más oclusões em escolares na Ilha do Governador, Rio de Janeiro: Parte 1. Classes I, II e II de Angle e mordida cruzada. Ortodontia, São Paulo, v.16, n.3, p.10-16, set. 1983.

SILVA FILHO, O.G.; FREITAS, S.F.; CAVASSAN, A.O. Prevalência de oclusão normal e má oclusão na dentadura mista em escolares da cidade de Bauru (São Paulo). R. APCD, São Paulo, v.43, n.3, p.287-290, dez. 1989.

Recebido em / Received: 20/06/2005 Aceito em / Accepted: 30/08/2005 